\title{
Infinite time interval backward stochastic differential equations with continuous coefficients
}

\author{
Zhaojun Zong ${ }^{\dagger}$ and Feng $\mathrm{Hu}^{*}+$
}

${ }^{*}$ Correspondence: hufengqf@163.com

'Zhaojun Zong and Feng

Hu contributed equally to

this work

School of Statistics,

Qufu Normal University,

Qufu 273165, China

\begin{abstract}
In this paper, we study the existence theorem for $L^{p}(1<p<2)$ solutions to a class of 1-dimensional infinite time interval backward stochastic differential equations (BSDEs) under the conditions that the coefficients are continuous and have linear growths. We also obtain the existence of a minimal solution. Furthermore, we study the existence and uniqueness theorem for $L^{p}(1<p<2)$ solutions of infinite time interval BSDEs with non-uniformly Lipschitz coefficients. It should be pointed out that the assumptions of this result is weaker than that of Theorem 3.1 in Zong (Turkish J Math 37:704718, 2013).
\end{abstract}

Keywords: Backward stochastic, Differential equation (BSDE), Linear growth condition, Comparison theorem

Mathematics Subject Classification: $60 \mathrm{H} 10$

\section{Background}

The theory of nonlinear backward stochastic differential equations (BSDEs for short) was developed by Pardoux and Peng (1990), from which we know that there exists a unique adapted and square integrable solution to a BSDE of the type

$$
y_{t}=\xi+\int_{t}^{T} g\left(s, y_{s}, z_{s}\right) \mathrm{d} s-\int_{t}^{T} z_{s} \mathrm{~d} W_{s}, \quad t \in[0, T],
$$

provided the function $g$ (also called the generator) is Lipschitz in both variables $y$ and $z$, and $\xi$ and $(g(t, 0,0))_{0 \leq t \leq T}$ are square integrable. The theory of BSDEs is very useful, due to the connection of this subject with mathematical finance, stochastic control, partial differential equation, stochastic game and stochastic geometry and mathematical economics. Later, many researchers developed the theory of BSDEs and their applications in a series of papers (for example, see Briand et al. (2003), Lepeltier and San Martin (1997), Pardoux (1997, 1998), Karoui et al. (1997) and the references therein) under some other assumptions on the coefficients but for a fixed terminal time $T>0$. Let us mention the contribution of Lepeltier and San Martin (1997). In Lepeltier and San Martin (1997), the authors got the existence of a solution for a 1-dimensional BSDE where the coefficient

(c) 2016 The Author(s). This article is distributed under the terms of the Creative Commons Attribution 4.0 International License (http://creativecommons.org/licenses/by/4.0/), which permits unrestricted use, distribution, and reproduction in any medium, provided you give appropriate credit to the original author(s) and the source, provide a link to the Creative Commons license, and indicate if changes were made. 
was continuous, it had linear growth, and the terminal condition was square integrable. They also obtained the existence of a minimal solution.

Chen and Wang (2000) obtained the existence and uniqueness theorem for $L^{2}$ solutions of BSDEs with non-uniformly Lipschitz coefficients when $T \equiv \infty$, by the martingale representation theorem and fixed point theorem. In fact, such a problem has been investigated by Peng (1990), Pardoux (1997), Darling and Pardoux (1997) and other researchers under the assumption that terminal value $\xi=0$ or $E\left[\mathrm{e}^{p \rho T}|\xi|^{p}\right]<\infty$ for some constant $\rho$ and random terminal time $T$ (i.e., $T$ is a stopping time). But in $L^{p}$ $(1<p<2)$, there is no the martingale representation theorem. Zong (2013) studied $L^{p}$ solutions to infinite time interval BSDEs with non-uniformly Lipschitz coefficients. She gave a new a priori estimate. By using this a priori estimate, she got the existence and uniqueness of $L^{p}$ solutions to infinite time interval BSDEs.

In this paper, we study the existence theorem for $L^{p}(1<p<2)$ solutions to a class of 1-dimensional infinite time interval BSDEs under the conditions that the coefficients are continuous and have linear growths. We also obtain the existence of a minimal solution. Furthermore, we study the existence and uniqueness theorem for $L^{p}(1<p<2)$ solutions of infinite time interval BSDEs with non-uniformly Lipschitz coefficients. It should be pointed out that the assumptions of this result is weaker than that of Theorem 3.1 in Zong (2013).

This paper is organized as follows. In "Preliminaries" section, we introduce some notations, assumptions and lemmas. In "Main results and proofs" section, we give our main results including the proofs.

\section{Preliminaries}

In this section, we shall present some notations, assumptions and lemmas that are used in this paper.

Notation. The Euclidean norm of a vector $x \in R^{k}$ will be denoted by $|x|$, and for a $k \times d$ matrix $A$, we define $\|A\|=\sqrt{\operatorname{Tr} A A^{*}}$, where $A^{*}$ is the transpose of $A$.

Let $(\Omega, \mathcal{F}, P)$ be a completed probability space, $\left(W_{t}\right)_{t \geq 0}$ be a $d$-dimensional standard Brownian motion defined on this space and $\left(\mathcal{F}_{t}\right)_{t \geq 0}$ be the natural filtration generated by Brownian motion $\left(W_{t}\right)_{t \geq 0}$, that is

$$
\mathcal{F}_{t}:=\sigma\left\{W_{s} ; s \leq t\right\} \vee \mathcal{N}
$$

where $\mathcal{N}$ is the set of all $P$-null subsets. Furthermore, we define $\mathcal{F}:=\sigma\left(\bigcup_{t \geq 0} \mathcal{F}_{t}\right)$.

We consider the following spaces:

$L^{p}\left(\Omega, \mathcal{F}, P, R^{k}\right):=\left\{\xi: \xi\right.$ is $R^{k}$-valued and $\mathcal{F}$-measurable random variable such that $\left.E\left[|\xi|^{p}\right]<\infty, p \geq 1\right\}$

$$
\begin{aligned}
& \mathcal{L}\left(\Omega, \mathcal{F}, P, R^{k}\right):=\bigcup_{p>1} L^{p}\left(\Omega, \mathcal{F}, P, R^{k}\right) ; \\
& \mathcal{S}^{p}\left(R^{k}\right):=\left\{V: V_{t} \text { is } R^{k} \text {-valued and } \mathcal{F}_{t} \text {-adapted } \quad \text { process } \quad\right. \text { such that } \\
& \left.E\left[\sup _{t \geq 0}\left|V_{t}\right|^{p}\right]<\infty, p \geq 1\right\} ; \\
& \mathcal{S}\left(R^{k}\right):=\bigcup_{p>1} \mathcal{S}^{p}\left(R^{k}\right) ; \\
& \mathcal{L}^{p}\left(R^{k \times d}\right):=\left\{V: V_{t} \text { is } R^{k \times d} \text {-valued and } \mathcal{F}_{t} \text {-adapted } \quad \text { process } \quad\right. \text { such that } \\
& \left.E\left[\left(\int_{0}^{\infty}\left\|V_{s}\right\|^{2} \mathrm{~d} s\right)^{\frac{p}{2}}\right]<\infty, p \geq 1\right\} ; \\
& \mathcal{L}\left(R^{k \times d}\right):=\bigcup_{p>1} \mathcal{L}^{p}\left(R^{k \times d}\right) .
\end{aligned}
$$

In the sequel, we assume that $1<p<2$. 
Consider the following infinite time interval BSDE

$$
Y_{t}=\xi+\int_{t}^{\infty} g\left(s, Y_{s}, Z_{s}\right) \mathrm{d} s-\int_{t}^{\infty} Z_{s} \mathrm{~d} W_{s}
$$

Let

$$
g: \Omega \times R_{+} \times R^{k} \times R^{k \times d} \mapsto R^{k}
$$

such that for any $(y, z) \in R^{k} \times R^{k \times d}, g(\cdot, y, z)$ is $\mathcal{F}_{t}$-progressively measurable. We make the following assumptions:

(A.1) There exist two positive non-random functions $\alpha(t)$ and $\beta(t)$, such that for all $y_{1}, y_{2} \in R^{k}, z_{1}, z_{2} \in R^{k \times d}$,

$$
\left|g\left(t, y_{1}, z_{1}\right)-g\left(t, y_{2}, z_{2}\right)\right| \leq \alpha(t)\left|y_{1}-y_{2}\right|+\beta(t)|| z_{1}-z_{2}||,
$$

where $\alpha(t)$ and $\beta(t)$ satisfy that $\int_{0}^{\infty} \alpha(t) \mathrm{d} t<\infty, \int_{0}^{\infty} \beta^{2}(t) \mathrm{d} t<\infty$;

(A.1') There exist two positive non-random functions $\alpha(t)$ and $\beta(t)$, such that for all $y_{1}, y_{2} \in R^{k}, z_{1}, z_{2} \in R^{k \times d}$,

$$
\left|g\left(t, y_{1}, z_{1}\right)-g\left(t, y_{2}, z_{2}\right)\right| \leq \alpha(t)\left|y_{1}-y_{2}\right|+\beta(t)|| z_{1}-z_{2}||,
$$

where $\alpha(t)$ and $\beta(t)$ satisfy that $\int_{0}^{\infty} \alpha(t) \mathrm{d} t<\infty, \int_{0}^{\infty} \beta(t) \mathrm{d} t<\infty, \int_{0}^{\infty} \beta^{2}(t) \mathrm{d} t<\infty$;

(A.2) $E\left[\left(\int_{0}^{\infty}|g(t, 0,0)| \mathrm{d} t\right)^{p}\right]<\infty$;

(A.2) There exists some constant $T \in[0, \infty)$ such that

$$
\begin{aligned}
& E\left[\left(\int_{0}^{T}|g(t, 0,0)| \mathrm{d} t\right)^{p}\right]<\infty, \\
& E\left[\left(\int_{T}^{\infty}|g(t, 0,0)| \mathrm{d} t\right)^{2}\right]<\infty ;
\end{aligned}
$$

(A.3) Linear growth: There exists a positive non-random function $\gamma(t)$ such that

$$
|g(\omega, t, y, z)| \leq \gamma(t)(1+|y|+|| z||), \quad \forall(\omega, t, y, z) \in \Omega \times R_{+} \times R^{k} \times R^{k \times d}
$$

where $\gamma(t)$ satisfies that $\int_{0}^{\infty} \gamma(t) \mathrm{d} t<\infty, \int_{0}^{\infty} \gamma^{2}(t) \mathrm{d} t<\infty$;

(A.4) For fixed $\omega$ and $t, g(\omega, t, \cdot, \cdot)$ is continuous.

Lemma 1 (see Zong 2013) Under assumptions (A.1') and (A.2'), if $\xi \in L^{p}\left(\Omega, \mathcal{F}, P, R^{k}\right)$, then $B S D E(2)$ has a unique solution $(Y, Z) \in \mathcal{S}^{p}\left(R^{k}\right) \times \mathcal{L}^{p}\left(R^{k \times d}\right)$.

\section{Main results and proofs}

In this section, first we study the existence and uniqueness theorem for $L^{p}$ solutions of infinite time interval BSDEs with non-uniformly Lipschitz coefficients. It should be pointed out that the assumptions of this result is weaker than that of Lemma 1.

Theorem 2 Under assumptions (A.1) and (A.2), if $\xi \in L^{p}\left(\Omega, \mathcal{F}, P, R^{k}\right)$, then BSDE (2) has a unique solution $(Y, Z) \in \mathcal{S}^{p}\left(R^{k}\right) \times \mathcal{L}^{p}\left(R^{k \times d}\right)$. 
In order to prove Theorem 2 , we give an a priori estimate.

Lemma 3 Suppose that (A.1) holds for g. Furthermore, each $\phi_{i}(i=1,2)$ satisfies that

$$
E\left[\left(\int_{0}^{\infty}\left|\phi_{i}(s)\right| \mathrm{d} s\right)^{p}\right]<\infty
$$

For any $T \in[0, \infty]$, let $\xi_{i} \in L^{p}\left(\Omega, \mathcal{F}_{T}, P, R^{k}\right),\left(Y^{i}, Z^{i}\right) \in \mathcal{S}^{p}\left(R^{k}\right) \times \mathcal{L}^{p}\left(R^{k \times d}\right)$ satisfy the following BSDEs:

$$
Y_{t}^{i}=\xi_{i}+\int_{t}^{T}\left[g\left(s, Y_{s}^{i}, Z_{s}^{i}\right)+\phi_{i}(s)\right] \mathrm{d} s-\int_{t}^{T} Z_{s}^{i} \mathrm{~d} W_{s}, \quad i=1,2 .
$$

Then there exists a positive constant $C_{p}$ depending only on $p$ such that, for any $\tau \in[0, T]$,

$$
\begin{aligned}
E\left[\sup _{s \in[\tau, T]}\left|Y_{s}^{1}-Y_{s}^{2}\right|^{p}+\left(\int_{\tau}^{T}|| Z_{s}^{1}-Z_{s}^{2}||^{2} \mathrm{~d} s\right)^{\frac{p}{2}}\right] \\
\leq C_{p} E\left[\left|\xi_{1}-\xi_{2}\right|^{p}+\left(\int_{0}^{\infty}\left|\phi_{1}(s)-\phi_{2}(s)\right| \mathrm{d} s\right)^{p}\right] \\
\quad+C_{p} l_{(\tau, T]} E\left[\sup _{s \in[\tau, T]}\left|Y_{s}^{1}-Y_{s}^{2}\right|^{p}+\left(\int_{\tau}^{T} \| Z_{s}^{1}-Z_{s}^{2}||^{2} \mathrm{~d} s\right)^{\frac{p}{2}}\right],
\end{aligned}
$$

where $l_{(\tau, T]}=\left(\int_{\tau}^{T} \alpha(s) \mathrm{d} s+\int_{\tau}^{T} \beta^{2}(s) \mathrm{d} s\right)^{\frac{p}{2}}+\left(\int_{\tau}^{T} \alpha(s) \mathrm{d} s\right)^{p}$.

Proof Applying Itô's formula to $\left|Y_{t}^{1}-Y_{t}^{2}\right|^{2}$, we have

$$
\begin{aligned}
\left|Y_{\tau}^{1}-Y_{\tau}^{2}\right|^{2}+\int_{\tau}^{T}\left\|Z_{s}^{1}-Z_{s}^{2}\right\|^{2} \mathrm{~d} s \\
=\left|\xi_{1}-\xi_{2}\right|^{2}+2 \int_{\tau}^{T}\left\langle Y_{s}^{1}-Y_{s}^{2},\left(g\left(s, Y_{s}^{1}, Z_{s}^{1}\right)-g\left(s, Y_{s}^{2}, Z_{s}^{2}\right)+\phi_{1}(s)-\phi_{2}(s)\right)\right\rangle \mathrm{d} s \\
\quad-2 \int_{\tau}^{T}\left\langle Y_{s}^{1}-Y_{s}^{2},\left(Z_{s}^{1}-Z_{s}^{2}\right) \mathrm{d} W_{s}\right\rangle
\end{aligned}
$$

From the Lipschitz assumption (A.1) on $g$, we have

$$
\begin{aligned}
2\langle & \left.Y_{s}^{1}-Y_{s}^{2},\left(g\left(s, Y_{s}^{1}, Z_{s}^{1}\right)-g\left(s, Y_{s}^{2}, Z_{s}^{2}\right)\right)\right\rangle \\
& \leq 2 \alpha(s)\left|Y_{s}^{1}-Y_{s}^{2}\right|^{2}+2 \beta(s)\left|Y_{s}^{1}-Y_{s}^{2}\right||| Z_{s}^{1}-Z_{s}^{2} \| \\
& \leq 2 \alpha(s)\left|Y_{s}^{1}-Y_{s}^{2}\right|^{2}+2 \beta^{2}(s)\left|Y_{s}^{1}-Y_{s}^{2}\right|^{2}+\frac{1}{2}\left\|Z_{s}^{1}-Z_{s}^{2}\right\|^{2} \\
& \leq 2\left(\alpha(s)+\beta^{2}(s)\right) \sup _{s \in[\tau, T]}\left|Y_{s}^{1}-Y_{s}^{2}\right|^{2}+\frac{1}{2}\left\|Z_{s}^{1}-Z_{s}^{2}\right\|^{2} .
\end{aligned}
$$


It follows that

$$
\begin{aligned}
& \frac{1}{2} \int_{\tau}^{T}|| Z_{s}^{1}-Z_{s}^{2}||^{2} \mathrm{~d} s \\
& \quad \leq\left|\xi_{1}-\xi_{2}\right|^{2}+2\left(\int_{\tau}^{T} \alpha(s) \mathrm{d} s+\int_{\tau}^{T} \beta^{2}(s) \mathrm{d} s\right) \sup _{s \in[\tau, T]}\left|Y_{s}^{1}-Y_{s}^{2}\right|^{2} \\
& \quad+2 \int_{\tau}^{T}\left|Y_{s}^{1}-Y_{s}^{2}\right|\left|\phi_{1}(s)-\phi_{2}(s)\right| \mathrm{d} s+2\left|\int_{\tau}^{T}\left\langle Y_{s}^{1}-Y_{s}^{2},\left(Z_{s}^{1}-Z_{s}^{2}\right) \mathrm{d} W_{s}\right\rangle\right| .
\end{aligned}
$$
Since $2 \int_{\tau}^{T}\left|Y_{s}^{1}-Y_{s}^{2}\right|\left|\phi_{1}(s)-\phi_{2}(s)\right| \mathrm{d} s \leq \sup _{s \in[\tau, T]}\left|Y_{s}^{1}-Y_{s}^{2}\right|^{2}+\left(\int_{0}^{\infty}\left|\phi_{1}(s)-\phi_{2}(s)\right| \mathrm{d} s\right)^{2}$,
we have

$$
\begin{aligned}
\int_{\tau}^{T} & \| Z_{s}^{1}-Z_{s}^{2}||^{2} \mathrm{~d} s \\
\leq & 4\left(\left|\xi_{1}-\xi_{2}\right|^{2}+\left(\int_{0}^{\infty}\left|\phi_{1}(s)-\phi_{2}(s)\right| \mathrm{d} s\right)^{2}\right) \\
& +4\left(1+\int_{\tau}^{T} \alpha(s) \mathrm{d} s+\int_{\tau}^{T} \beta^{2}(s) \mathrm{d} s\right) \sup _{s \in[\tau, T]}\left|Y_{s}^{1}-Y_{s}^{2}\right|^{2} \\
& +4\left|\int_{\tau}^{T}\left\langle Y_{s}^{1}-Y_{s}^{2},\left(Z_{s}^{1}-Z_{s}^{2}\right) \mathrm{d} W_{s}\right\rangle\right| .
\end{aligned}
$$

Using the following fact: if $b, a_{i} \geq 0$ and $b \leq \sum_{i=1}^{n} a_{i}$, then $b^{p} \leq \sum_{i=1}^{n} a_{i}^{p}$ for any $p \in(0,1)$, we have

$$
\begin{aligned}
& \left(\int_{\tau}^{T}|| Z_{s}^{1}-Z_{s}^{2}||^{2} \mathrm{~d} s\right)^{\frac{p}{2}} \\
& \leq c_{p}\left(\left|\xi_{1}-\xi_{2}\right|^{p}+\left(\int_{0}^{\infty}\left|\phi_{1}(s)-\phi_{2}(s)\right| \mathrm{d} s\right)^{p}\right) \\
& \quad+c_{p}\left[1+\left(\int_{\tau}^{T} \alpha(s) \mathrm{d} s+\int_{\tau}^{T} \beta^{2}(s) \mathrm{d} s\right)^{\frac{p}{2}}\right] \sup _{s \in[\tau, T]}\left|Y_{s}^{1}-Y_{s}^{2}\right|^{p} \\
& \quad+c_{p}\left(\left|\int_{\tau}^{T}\left(Y_{s}^{1}-Y_{s}^{2}\right)\left(Z_{s}^{1}-Z_{s}^{2}\right) \mathrm{d} W_{s}\right|^{\frac{p}{2}}\right)
\end{aligned}
$$

where $c_{p}$ is a positive constant depending only on $p$. By the Burkholder-Davis-Gundy inequality, we get

$$
\begin{aligned}
c_{p} E\left[\left|\int_{\tau}^{T}\left\langle Y_{s}^{1}-Y_{s}^{2},\left(Z_{s}^{1}-Z_{s}^{2}\right) \mathrm{d} W_{s}\right\rangle\right|^{\frac{p}{2}}\right] \\
\leq d_{p} E\left[\left(\int_{\tau}^{T}\left|Y_{s}^{1}-Y_{s}^{2}\right|^{2} \| Z_{s}^{1}-\left.Z_{s}^{2}\right|^{2} \mathrm{~d} s\right)^{\frac{p}{4}}\right] \\
\leq d_{p} E\left[\sup _{s \in[\tau, T]}\left|Y_{s}^{1}-Y_{s}^{2}\right|^{\frac{p}{2}}\left(\int_{\tau}^{T}\left\|Z_{s}^{1}-Z_{s}^{2}\right\|^{2} \mathrm{~d} s\right)^{\frac{p}{4}}\right]
\end{aligned}
$$


and thus

$$
\begin{aligned}
c_{p} E\left[\left|\int_{\tau}^{T}\left\langle Y_{s}^{1}-Y_{s}^{2},\left(Z_{s}^{1}-Z_{s}^{2}\right) \mathrm{d} W_{s}\right\rangle\right|^{\frac{p}{2}}\right] \leq & \frac{1}{2} E\left[\left(\int_{\tau}^{T}\left\|Z_{s}^{1}-Z_{s}^{2}\right\|^{2} \mathrm{~d} s\right)^{\frac{p}{2}}\right] \\
& +\frac{d_{p}^{2}}{2} E\left[\sup _{s \in[\tau, T]}\left|Y_{s}^{1}-Y_{s}^{2}\right|^{p}\right],
\end{aligned}
$$

where $d_{p}$ is a positive constant depending only on $p$. From (8) and (10), we have

$$
\begin{aligned}
E\left[\left(\int_{\tau}^{T} \| Z_{s}^{1}-Z_{s}^{2}||^{2} \mathrm{~d} s\right)^{\frac{p}{2}}\right] \\
\leq C\left(E\left[\left|\xi_{1}-\xi_{2}\right|^{p}\right]+E\left[\left(\int_{0}^{\infty}\left|\phi_{1}(s)-\phi_{2}(s)\right| \mathrm{d} s\right)^{p}\right]\right) \\
+C\left(1+l_{(\tau, T]}\right) E\left[\sup _{s \in[\tau, T]}\left|Y_{s}^{1}-Y_{s}^{2}\right|^{p}\right],
\end{aligned}
$$

where $C$ is a positive constant depending only on $p$.

On the other hand, we prove

$$
\begin{aligned}
E\left[\sup _{s \in[\tau, T]}\left|Y_{s}^{1}-Y_{s}^{2}\right|^{p}\right] & \\
\leq & C^{\prime} E\left[\left|\xi_{1}-\xi_{2}\right|^{p}+\left(\int_{0}^{\infty}\left|\phi_{1}(s)-\phi_{2}(s)\right| \mathrm{d} s\right)^{p}\right] \\
& +C^{\prime} l_{(\tau, T]} E\left[\sup _{s \in[\tau, T]}\left|Y_{s}^{1}-Y_{s}^{2}\right|^{p}+\left(\int_{\tau}^{T}\left\|Z_{s}^{1}-Z_{s}^{2}\right\|^{2} \mathrm{~d} s\right)^{\frac{p}{2}}\right],
\end{aligned}
$$

where $C^{\prime}$ is a positive constant depending only on $p$. Obviously, $\left\{\int_{\tau}^{t}\left(Z_{s}^{1}-Z_{s}^{2}\right) \mathrm{d} W_{s} ; \tau \leq t \leq T\right\}$ is an $\mathcal{F}_{t}$-martingale. Thus, it follows that

$$
Y_{t}^{1}-Y_{t}^{2}=E\left[\left(\xi_{1}-\xi_{2}\right)+\int_{t}^{T}\left(g\left(s, Y_{s}^{1}, Z_{s}^{1}\right)-g\left(s, Y_{s}^{2}, Z_{s}^{2}\right)+\phi_{1}(s)-\phi_{2}(s)\right) \mathrm{d} s \mid \mathcal{F}_{t}\right] .
$$

Applying Doob's inequality, we can deduce that

$$
\begin{aligned}
E & {\left[\sup _{t \in[\tau, T]}\left|Y_{t}^{1}-Y_{t}^{2}\right|^{p}\right] } \\
& \leq E\left[\sup _{t \in[\tau, T]}\left(E\left[\left|\xi_{1}-\xi_{2}\right|+\int_{\tau}^{T}\left|g\left(s, Y_{s}^{1}, Z_{s}^{1}\right)-g\left(s, Y_{s}^{2}, Z_{s}^{2}\right)+\phi_{1}(s)-\phi_{2}(s)\right| \mathrm{d} s \mid \mathcal{F}_{t}\right]\right)^{p}\right] \\
& \leq\left(\frac{p}{p-1}\right)^{p} E\left[\left(\left|\xi_{1}-\xi_{2}\right|+\int_{\tau}^{T}\left|g\left(s, Y_{s}^{1}, Z_{s}^{1}\right)-g\left(s, Y_{s}^{2}, Z_{s}^{2}\right)+\phi_{1}(s)-\phi_{2}(s)\right| \mathrm{d} s\right)^{p}\right] \\
& \leq D_{p} E\left[\left|\xi_{1}-\xi_{2}\right|^{p}+\left(\int_{0}^{\infty}\left|\phi_{1}(s)-\phi_{2}(s)\right| \mathrm{d} s\right)^{p}+\left(\int_{\tau}^{T}\left|g\left(s, Y_{s}^{1}, Z_{s}^{1}\right)-g\left(s, Y_{s}^{2}, Z_{s}^{2}\right)\right| \mathrm{d} s\right)^{p}\right]
\end{aligned}
$$


where $D_{p}$ is a positive constant depending only on $p$. From the Lipschitz assumption (A.1) on $g$, we have

$$
\begin{aligned}
E\left[\left(\int_{\tau}^{T}\left|g\left(s, Y_{s}^{1}, Z_{s}^{1}\right)-g\left(s, Y_{s}^{2}, Z_{s}^{2}\right)\right| \mathrm{d} s\right)^{p}\right] \\
\leq E\left[\left(\int_{\tau}^{T}\left(\alpha(s)\left|Y_{s}^{1}-Y_{s}^{2}\right|+\beta(s)|| Z_{s}^{1}-Z_{s}^{1}||\right) \mathrm{d} s\right)^{p}\right] \\
\leq M_{p}\left(\int_{\tau}^{T} \alpha(s) \mathrm{d} s\right)^{p} E\left[\sup _{s \in[\tau, T]}\left|Y_{s}^{1}-Y_{s}^{2}\right|^{p}\right] \\
\quad+M_{p}\left(\int_{\tau}^{T} \beta^{2}(s) \mathrm{d} s\right)^{\frac{p}{2}} E\left[\left(\int_{\tau}^{T}\left\|Z_{s}^{1}-Z_{s}^{2}\right\|^{2} \mathrm{~d} s\right)^{\frac{p}{2}}\right],
\end{aligned}
$$

where $M_{p}$ is a positive constant depending only on $p$. From (14) and (15), we have

$$
\begin{aligned}
E\left[\sup _{s \in[\tau, T]}\left|Y_{s}^{1}-Y_{s}^{2}\right|^{p}\right] & \\
\leq & C^{\prime} E\left[\left|\xi_{1}-\xi_{2}\right|^{p}+\left(\int_{0}^{\infty}\left|\phi_{1}(s)-\phi_{2}(s)\right| \mathrm{d} s\right)^{p}\right] \\
& +C^{\prime} l_{(\tau, T]} E\left[\sup _{s \in[\tau, T]}\left|Y_{s}^{1}-Y_{s}^{2}\right|^{p}+\left(\int_{\tau}^{T}\left\|Z_{s}^{1}-Z_{s}^{2}\right\|^{2} \mathrm{~d} s\right)^{\frac{p}{2}}\right],
\end{aligned}
$$

where $C^{\prime}$ is a positive constant depending only on $p$.

Combining (11) with (16), we get

$$
\begin{aligned}
E\left[\sup _{s \in[\tau, T]}\left|Y_{s}^{1}-Y_{s}^{2}\right|^{p}+\left(\int_{\tau}^{T}\left|Z_{s}^{1}-Z_{s}^{2}\right|^{2} \mathrm{~d} s\right)^{\frac{p}{2}}\right] \\
\leq C_{p} E\left[\left|\xi_{1}-\xi_{2}\right|^{p}+\left(\int_{0}^{\infty}\left|\phi_{1}(s)-\phi_{2}(s)\right| \mathrm{d} s\right)^{p}\right] \\
\quad+C_{p} l_{(\tau, T]} E\left[\sup _{s \in[\tau, T]}\left|Y_{s}^{1}-Y_{s}^{2}\right|^{p}+\left(\int_{\tau}^{T}|| Z_{s}^{1}-Z_{s}^{2}||^{2} \mathrm{~d} s\right)^{\frac{p}{2}}\right],
\end{aligned}
$$

where $C_{p}$ is a positive constant depending only on $p$. The proof of Lemma 3 is complete.

Proof of Theorem 2 Let $\xi^{n}:=(\xi \wedge n) \vee(-n)$ and $g_{n}(t, y, z):=g(t, y, z)-g(t, 0,0)$ $+h_{n}(g(t, 0,0))$, where $h_{n}(g(t, 0,0)):=\frac{g(t, 0,0) n e^{-t}}{|g(t, 0,0)| \vee\left(n e^{-t}\right)}$. By Theorem 1.2 in Chen and Wang (2000), BSDE

$$
Y_{t}^{n}=\xi^{n}+\int_{t}^{\infty} g_{n}\left(s, Y_{s}^{n}, Z_{s}^{n}\right) \mathrm{d} s-\int_{t}^{\infty} Z_{s}^{n} \mathrm{~d} W_{s}
$$

has a unique solution $\left(Y^{n}, Z^{n}\right) \in \mathcal{S}^{2}\left(R^{k}\right) \times \mathcal{L}^{2}\left(R^{k \times d}\right)$. Since 


$$
\left(\int_{0}^{\infty} \alpha(s) \mathrm{d} s+\int_{0}^{\infty} \beta^{2}(s) \mathrm{d} s\right)^{\frac{p}{2}}+\left(\int_{0}^{\infty} \alpha(s) \mathrm{d} s\right)^{p}<\infty,
$$

we can choose a strictly increasing sequence $0=t_{0}<t_{1}<\cdots<t_{N}<t_{N+1}=\infty$, such that

$$
l_{\left(t_{i}, t_{i+1}\right]} \leq \frac{1}{2 C_{p}}, \quad i=0,1,2, \ldots, N .
$$

Applying Lemma 3, we have

$$
\begin{aligned}
E\left[\sup _{s \in\left[t_{i}, t_{i+1}\right]}\left|Y_{s}^{m+n}-Y_{s}^{n}\right|^{p}+\left(\int_{t_{i}}^{t_{i+1}}|| Z_{s}^{m+n}-Z_{s}^{n}||^{2} \mathrm{~d} s\right)^{\frac{p}{2}}\right] \\
\leq C_{p} E\left[\left|Y_{t_{i+1}}^{m+n}-Y_{t_{i+1}}^{n}\right|^{p}\right] \\
\quad+C_{p} E\left[\left(\int_{0}^{\infty}\left|h_{n+m}(g(s, 0,0))-h_{n}(g(s, 0,0))\right| \mathrm{d} s\right)^{p}\right] \\
\quad+\frac{1}{2} E\left[\sup _{s \in\left[t_{i}, t_{i+1}\right]}\left|Y_{s}^{m+n}-Y_{s}^{n}\right|^{p}+\left(\int_{t_{i}}^{t_{i+1}}|| Z_{s}^{m+n}-Z_{s}^{n}||^{2} \mathrm{~d} s\right)^{\frac{p}{2}}\right] .
\end{aligned}
$$

Thus

$$
\begin{aligned}
& E\left[\sup _{s \in\left[t_{i}, t_{i+1}\right]}\left|Y_{s}^{m+n}-Y_{s}^{n}\right|^{p}+\left(\int_{t_{i}}^{t_{i+1}}|| Z_{s}^{m+n}-Z_{s}^{n}||^{2} \mathrm{~d} s\right)^{\frac{p}{2}}\right] \\
& \leq 2 C_{p} E\left[\left|Y_{t_{i+1}}^{m+n}-Y_{t_{i+1}}^{n}\right|^{p}\right] \\
&+2 C_{p} E\left[\left(\int_{0}^{\infty}\left|h_{n+m}(g(s, 0,0))-h_{n}(g(s, 0,0))\right| \mathrm{d} s\right)^{p}\right] \\
& \leq 2 C_{p} E\left[\sup _{s \in\left[t_{i+1}, t_{i+2}\right]}\left|Y_{s}^{m+n}-Y_{s}^{n}\right|^{p}+\left(\int_{t_{i+1}}^{t_{i+2}}|| Z_{s}^{m+n}-Z_{s}^{n}||^{2} \mathrm{~d} s\right)^{\frac{p}{2}}\right] \\
&+2 C_{p} E\left[\left(\int_{0}^{\infty}\left|h_{n+m}(g(s, 0,0))-h_{n}(g(s, 0,0))\right| \mathrm{d} s\right)^{p}\right], \quad i=0,1,2, \ldots, N-1 .
\end{aligned}
$$

In particulary, we have

$$
\begin{aligned}
E\left[\sup _{s \geq t_{N}}\left|Y_{s}^{m+n}-Y_{s}^{n}\right|^{p}+\left(\int_{t_{N}}^{\infty} \| Z_{s}^{m+n}-Z_{s}^{n}||^{2} \mathrm{~d} s\right)^{\frac{p}{2}}\right] \\
\quad \leq 2 C_{p} E\left[\left|\xi^{m+n}-\xi^{n}\right|^{p}\right] \\
\quad+2 C_{p} E\left[\left(\int_{0}^{\infty}\left|h_{n+m}(g(s, 0,0))-h_{n}(g(s, 0,0))\right| \mathrm{d} s\right)^{p}\right] .
\end{aligned}
$$

From (19) and (20), it follows that 


$$
\begin{aligned}
& E\left[\sup _{s \geq 0}\left|Y_{s}^{n+m}-Y_{s}^{n}\right|^{p}+\left(\int_{0}^{\infty}|| Z_{s}^{n+m}-Z_{s}^{n}||^{2} \mathrm{~d} s\right)^{\frac{p}{2}}\right] \\
& \leq \sum_{i=0}^{N} E\left[\sup _{s \in\left[t_{i}, t_{i+1}\right]}\left|Y_{s}^{m+n}-Y_{s}^{n}\right|^{p}+\left(\int_{t_{i}}^{t_{i+1}}|| Z_{s}^{m+n}-\left.Z_{s}^{n}\right|^{2} \mathrm{~d} s\right)^{\frac{p}{2}}\right] \\
& \leq\left(2 C_{p}+\left(2 C_{p}\right)^{2}+\cdots+\left(2 C_{p}\right)^{N+1}\right) E\left[\left|\xi^{m+n}-\xi^{n}\right|^{p}\right] \\
&+(N+1)\left(2 C_{p}+\left(2 C_{p}\right)^{2}+\cdots+\left(2 C_{p}\right)^{N+1}\right) E\left[\left(\int_{0}^{\infty}\left|h_{n+m}(g(s, 0,0))-h_{n}(g(s, 0,0))\right| \mathrm{d} s\right)^{p}\right] \\
& \leq \bar{C} E\left[\left|\xi^{m+n}-\xi^{n}\right|^{p}\right] \\
&+\bar{C} E\left[\left(\int_{0}^{\infty}\left|h_{n+m}(g(s, 0,0))-h_{n}(g(s, 0,0))\right| \mathrm{d} s\right)^{p}\right],
\end{aligned}
$$

where $\bar{C}=(N+1)\left(2 C_{p}+\left(2 C_{p}\right)^{2}+\cdots+\left(2 C_{p}\right)^{N+1}\right)$. The right-hand side of Inequality (21) clearly tends to 0 , as $n \rightarrow \infty$, uniformly in $m$, so we have a Cauchy sequence and the limit is a solution to $\operatorname{BSDE}(2)$. Let us consider $(Y, Z)$ and $\left(Y^{\prime}, Z^{\prime}\right)$ to be two solutions to BSDE (2). In a similar manner to the proof of Inequality (21), we can obtain

$$
E\left[\sup _{s \geq 0}\left|Y_{s}-Y_{s}^{\prime}\right|^{p}+\left(\int_{0}^{\infty}\left\|Z_{s}-Z_{s}^{\prime}\right\|^{2} \mathrm{~d} s\right)^{\frac{p}{2}}\right] \leq 0 .
$$

Thus, we get immediately $(Y, Z)=\left(Y^{\prime}, Z^{\prime}\right)$. The proof of Theorem 2 is complete.

Theorem 4 (Comparison Theorem) Assume that $k=1$. We make the same assumptions on $\xi, g$ and $\bar{\xi}, \bar{g}$ as in Theorem 2. Let $(\bar{Y}, \bar{Z})$ be a solution of BSDE

$$
\bar{Y}_{t}=\bar{\xi}+\int_{t}^{\infty} \bar{g}\left(s, \bar{Y}_{s}, \bar{Z}_{s}\right) \mathrm{d} s-\int_{t}^{\infty} \bar{Z}_{s} \mathrm{~d} W_{s} .
$$

If we suppose that:

$$
\hat{\xi}:=\xi-\bar{\xi} \leq 0, \quad \hat{g}_{t}:=g\left(t, \bar{Y}_{t}, \bar{Z}_{t}\right)-\bar{g}\left(t, \bar{Y}_{t}, \bar{Z}_{t}\right) \leq 0, \text { a.s. }
$$

then

$$
\hat{Y}_{t}:=Y_{t}-\bar{Y}_{t} \leq 0, \text { a.s., } \forall t \in[0, \infty) .
$$

Moreover, $\bar{Y}_{t}=Y_{t}$ a.s., if and only if $\bar{\xi}=\xi$ a.s., $\bar{g}\left(t, Y_{t}, Z_{t}\right)=g\left(t, Y_{t}, Z_{t}\right)$ a.s..

Proof Suppose that $W_{t}=\left(W_{t}^{1}, W_{t}^{2}, \ldots, W_{t}^{d}\right)^{T}, \forall t \in[0, \infty)$, where $W_{t}^{i}$ is the $i$ th components of $W_{t}$. Let us consider the following BSDEs

$$
\begin{gathered}
Y_{t}=\xi+\int_{t}^{\infty} g\left(s, Y_{s}, Z_{s}\right) \mathrm{d} s-\int_{t}^{\infty} Z_{s} \mathrm{~d} W_{s} \\
\bar{Y}_{t}=\bar{\xi}+\int_{t}^{\infty} \bar{g}\left(s, \bar{Y}_{s}, \bar{Z}_{s}\right) \mathrm{d} s-\int_{t}^{\infty} \bar{Z}_{s} \mathrm{~d} W_{s}
\end{gathered}
$$


where $Z_{t}=\left(Z_{t}^{1}, Z_{t}^{2}, \ldots, Z_{t}^{d}\right)^{T}, \bar{Z}_{t}=\left(\bar{Z}_{t}^{1}, \bar{Z}_{t}^{2}, \ldots, \bar{Z}_{t}^{d}\right)^{T}, \quad \forall t \in[0, \infty)$ and $\int_{t}^{\infty} Z_{s} \mathrm{~d} W_{s}$ $=\sum_{i=1}^{d} \int_{t}^{\infty} Z_{t}^{i} \mathrm{~d} W_{t}^{i}, \int_{t}^{\infty} \bar{Z}_{s} \mathrm{~d} W_{s}=\sum_{i=1}^{d} \int_{t}^{\infty} \bar{Z}_{t}^{i} \mathrm{~d} W_{t}^{i}$. Then, we have

$$
\hat{Y}_{t}=\hat{\xi}+\int_{t}^{\infty}\left(a_{s} \hat{Y}_{s}+\left\langle b_{s}, \hat{Z}_{s}\right\rangle+\hat{g}_{s}\right) \mathrm{d} s-\int_{t}^{\infty} \hat{Z}_{s} \mathrm{~d} W_{s}
$$

where

$$
\begin{aligned}
\hat{Z}_{s} & =Z_{s}-\bar{Z}_{s}=\left(Z_{t}^{1}-\bar{Z}_{t}^{1}, Z_{t}^{2}-\bar{Z}_{t}^{2}, \ldots, Z_{t}^{d}-\bar{Z}_{t}^{d}\right)^{T}, \\
Z_{s}^{(i)} & =\left(\bar{Z}_{s}^{1}, \ldots, \bar{Z}_{s}^{i}, Z_{s}^{i+1}, \ldots, Z_{s}^{d}\right)^{T}, \quad i=1,2, \ldots, d-1, \\
Z_{s}^{(0)} & =Z_{s}=\left(Z_{s}^{1}, Z_{s}^{2}, \ldots, Z_{s}^{d}\right)^{T}, \\
Z_{s}^{(d)} & =\bar{Z}_{s}=\left(\bar{Z}_{s}^{1}, \bar{Z}_{s}^{2}, \ldots, \bar{Z}_{s}^{d}\right)^{T}, \\
a_{s} & =\frac{g\left(s, Y_{s}, Z_{s}\right)-g\left(s, \bar{Y}_{s}, Z_{s}\right)}{\hat{Y}_{s}} 1_{\left\{\hat{Y}_{s} \neq 0\right\}}, \\
b_{s}^{i} & =\frac{g\left(s, \bar{Y}_{s}, Z_{s}^{(i-1)}\right)-g\left(s, \bar{Y}_{s}, Z_{s}^{(i)}\right)}{Z_{s}^{i}-\bar{Z}_{s}^{i}} 1_{\left\{Z_{s}^{i}-\bar{Z}_{s}^{i} \neq 0\right\}}, \quad i=1,2, \ldots, d, \\
b_{s} & =\left(b_{s}^{1}, b_{s}^{2}, \ldots, b_{s}^{d}\right)^{T},
\end{aligned}
$$

which imply $\left|a_{s}\right| \leq \alpha(s),\left|b_{s}\right| \leq \beta(s)$.

Solving (23), we know that the unique solution of BSDEs (23) can be represented as

$$
\hat{Y}_{t}=E\left[\hat{\xi} X_{\infty}+\int_{t}^{\infty} \hat{g}_{s} X_{s} \mathrm{~d} s \mid \mathcal{F}_{t}\right]
$$

where

$$
X_{s}=\exp \left[\int_{t}^{s}\left(a_{r}-\frac{1}{2}\left|b_{r}\right|^{2}\right) \mathrm{d} r+\int_{t}^{s} b_{r} \mathrm{~d} W_{r}\right], \quad s \geq t .
$$

From (24), we can obtain $\hat{Y}_{t} \leq 0$, a.s. and if $\bar{\xi}=\xi$ a.s., $\bar{g}\left(t, Y_{t}, Z_{t}\right)=g\left(t, Y_{t}, Z_{t}\right)$ a.s., then $\bar{Y}_{t}=Y_{t}$ a.s..

Choosing $t=0$ in (24) and from the strict monotonicity of $E[\cdot]$, we can obtain that if $\bar{Y}_{0}=Y_{0}$, then $\bar{\xi}=\xi$ a.s., $\bar{g}\left(t, Y_{t}, Z_{t}\right)=g\left(t, Y_{t}, Z_{t}\right)$ a.s.. The proof of Theorem 4 is complete.

Now we prove the existence theorem for $L^{p}$ solutions of 1-dimensional infinite time interval BDSDEs which generalizes Theorem 1 in Lepeltier and San Martin (1997).

Theorem 5 Assume that $k=1$. Under assumptions (A.3) and (A.4), if $\xi \in L^{p}(\Omega, \mathcal{F}, P, R)$, then $\operatorname{BSDE}(2)$ has a solution $(Y, Z) \in \mathcal{S}^{p}(R) \times \mathcal{L}^{p}\left(R^{d}\right)$. Also, there is a minimal solution $(\underline{Y}, \underline{Z})$ of $B S D E(2)$, in the sense that for any other solution $(Y, Z)$ of $(2)$, we have $\underline{Y} \leq Y$.

In order to prove Theorem 5 , we need the following lemmas.

Lemma 6 Suppose that (A.3) and (A.4) hold forg. For each $(\omega, t, y, z) \in \Omega \times R_{+} \times R \times R^{d}$, define the sequence of functions 


$$
g_{n}(\omega, t, y, z):=\inf _{\left(y^{\prime}, z^{\prime}\right) \in Q}\left\{g\left(\omega, t, y^{\prime}, z^{\prime}\right)+n \gamma(t)\left(\left|y-y^{\prime}\right|+\left|z-z^{\prime}\right|\right)\right\},
$$

where $Q$ is the set of all rational numbers in $R^{d+1}$. Then $g_{n}$ satisfies

(i) Linear growth: $\forall(\omega, t, y, z) \in \Omega \times R_{+} \times R \times R^{d},\left|g_{n}(\omega, t, y, z)\right| \leq \gamma(t)(1+|y|+|z|)$;

(ii) Monotonicity in $n: \forall(\omega, t, y, z) \in \Omega \times R_{+} \times R \times R^{d}, g_{n}(\omega, t, y, z) \uparrow$;

(iii) Lipschitz condition: $\forall(\omega, t, y, z),\left(\omega, t, y^{\prime}, z^{\prime}\right) \in \Omega \times R_{+} \times R \times R^{d}$,

$$
\left|g_{n}(\omega, t, y, z)-g_{n}\left(\omega, t, y^{\prime}, z^{\prime}\right)\right| \leq n \gamma(t)\left(\left|y-y^{\prime}\right|+\left|z-z^{\prime}\right|\right) ;
$$

(iv) Strong convergence: if $\left(y_{n}, z_{n}\right) \rightarrow(y, z)$, as $n \rightarrow \infty$, then

$$
g_{n}\left(\omega, t, y_{n}, z_{n}\right) \rightarrow g(\omega, t, y, z), \text { as } n \rightarrow \infty .
$$

The proof of Lemma 6 is very similar to that of Lemma 1 in Lepeltier and San Martin (1997), so we omit it.

We also define the function

$$
G(\omega, t, y, z):=\gamma(t)(1+|y|+|z|), \quad \forall(\omega, t, y, z) \in \Omega \times R_{+} \times R \times R^{d} .
$$

For each given $\xi \in L^{p}(\Omega, \mathcal{F}, P, R)$, by Theorem 2, there exist two pair of processes $\left(Y^{n}, Z^{n}\right)$ and $(U, V)$, which are the solutions to the following BSDEs

$$
\begin{aligned}
& Y_{t}^{n}=\xi+\int_{t}^{\infty} g_{n}\left(s, Y_{s}^{n}, Z_{s}^{n}\right) \mathrm{d} s-\int_{t}^{\infty} Z_{s}^{n} \mathrm{~d} W_{s}, \\
& U_{t}=\xi+\int_{t}^{\infty} G\left(s, U_{s}, V_{s}\right) \mathrm{d} s-\int_{t}^{\infty} V_{s} \mathrm{~d} W_{s}
\end{aligned}
$$

respectively. From Theorem 4 and Lemma 6, we get

$$
\forall n \geq m, \quad Y^{m} \leq Y^{n} \leq U \text {, a.s. }
$$

Lemma 7 There exists a constant $A>0$ independent of $n$, such that

$$
\begin{aligned}
& E\left[\sup _{t \geq 0}\left|U_{t}\right|^{p}\right] \leq A, \quad E\left[\left(\int_{0}^{\infty}\left|V_{t}\right|^{2} \mathrm{~d} t\right)^{\frac{p}{2}}\right] \leq A, \\
& E\left[\sup _{t \geq 0}\left|Y_{t}^{n}\right|^{p}\right] \leq A, \quad E\left[\left(\int_{0}^{\infty}\left|Z_{t}^{n}\right|^{2} \mathrm{~d} t\right)^{\frac{p}{2}}\right] \leq A, \quad \forall n \in N .
\end{aligned}
$$

Proof Since $(U, V)$ is the solution of BSDE (26), there exists a constant $B>0$ independent of $n$, such that

$$
E\left[\sup _{t \geq 0}\left|U_{t}\right|^{p}\right] \leq B, \quad E\left[\left(\int_{0}^{\infty}\left|V_{t}\right|^{2} \mathrm{~d} t\right)^{\frac{p}{2}}\right] \leq B .
$$

From Inequality (27), we can obtain that for each $n \in N$, 


$$
\left|Y_{t}^{n}\right|^{p} \leq 2^{p-1}\left(\left|Y_{t}^{1}\right|^{p}+\left|U_{t}\right|^{p}\right) .
$$

Thus, there exists a constant $C>0$ independent of $n$, such that

$$
E\left[\sup _{t \geq 0}\left|Y_{t}^{n}\right|^{p}\right] \leq C, \forall n \in N .
$$

At last, we prove the boundedness of $E\left[\left(\int_{0}^{\infty}\left|Z_{t}^{n}\right|^{2} \mathrm{~d} t\right)^{\frac{p}{2}}\right]$. Applying Itô's formula to $\left|Y_{t}^{n}\right|^{2}$, we have

$$
\begin{aligned}
& \left|Y_{0}^{n}\right|^{2}+\int_{0}^{\infty}\left|Z_{t}^{n}\right|^{2} \mathrm{~d} t \\
& \quad=|\xi|^{2}+2 \int_{0}^{\infty} Y_{t}^{n} g_{n}\left(t, Y_{t}^{n}, Z_{t}^{n}\right) \mathrm{d} t-2 \int_{0}^{\infty} Y_{t}^{n} Z_{t}^{n} \mathrm{~d} W_{t} .
\end{aligned}
$$

By Lemma 6 (i), we know $\left|g_{n}(t, y, z)\right| \leq \gamma(t)(1+|y|+|z|)$. Thus, we have

$$
\begin{aligned}
2\left|Y_{t}^{n} g_{n}\left(t, Y_{t}^{n}, Z_{t}^{n}\right)\right| \leq & 2 \gamma(t)\left(\left|Y_{t}^{n}\right|+\left|Y_{t}^{n}\right|^{2}+\left|Y_{t}^{n} Z_{t}^{n}\right|\right) \\
\leq & \gamma(t)\left(1+\left|Y_{t}^{n}\right|^{2}\right)+2 \gamma(t)\left|Y_{t}^{n}\right|^{2} \\
& +2 \gamma^{2}(t)\left|Y_{t}^{n}\right|^{2}+\frac{1}{2}\left|Z_{t}^{n}\right|^{2} \\
\leq & \gamma(t)+3\left(\gamma(t)+\gamma^{2}(t)\right) \sup _{t \geq 0}\left|Y_{t}^{n}\right|^{2}+\frac{1}{2}\left|Z_{t}^{n}\right|^{2}
\end{aligned}
$$

It follows that

$$
\begin{aligned}
\int_{0}^{\infty}\left|Z_{t}^{n}\right|^{2} \mathrm{~d} t \leq 2|\xi|^{2} & +2 \int_{0}^{\infty} \gamma(t) \mathrm{d} t \\
& +6\left(\int_{0}^{\infty} \gamma(t) \mathrm{d} t+\int_{0}^{\infty} \gamma^{2}(t) \mathrm{d} t\right) \sup _{t \geq 0}\left|Y_{t}^{n}\right|^{2}+4\left|\int_{0}^{\infty} Y_{t}^{n} Z_{t}^{n} \mathrm{~d} W_{t}\right| .
\end{aligned}
$$

Using the following fact: if $b, a_{i} \geq 0$ and $b \leq \sum_{i=1}^{n} a_{i}$, then $b^{p} \leq \sum_{i=1}^{n} a_{i}^{p}$ for any $p \in(0,1)$, we have

$$
\begin{aligned}
\left(\int_{0}^{\infty}\left|Z_{t}^{n}\right|^{2} \mathrm{~d} t\right)^{\frac{p}{2}} \leq & c_{p}\left(|\xi|^{p}+\left(\int_{0}^{\infty} \gamma(t) \mathrm{d} t\right)^{\frac{p}{2}}\right) \\
& +c_{p}\left(\int_{0}^{\infty} \gamma(t) \mathrm{d} t+\int_{0}^{\infty} \gamma^{2}(t) \mathrm{d} t\right)^{\frac{p}{2}} \\
& \sup _{t \geq 0}\left|Y_{t}^{n}\right|^{p}+c_{p}\left|\int_{0}^{\infty} Y_{t}^{n} Z_{t}^{n} \mathrm{~d} W_{t}\right|^{\frac{p}{2}}
\end{aligned}
$$

where $c_{p}$ is a positive constant depending only on $p$. By the Burkholder-Davis-Gundy inequality, we get 


$$
\begin{aligned}
c_{p} E\left[\left|\int_{0}^{\infty} Y_{t}^{n} Z_{t}^{n} \mathrm{~d} W_{t}\right|^{\frac{p}{2}}\right] & \leq d_{p} E\left[\left(\int_{0}^{\infty}\left|Y_{t}^{n} Z_{t}^{n}\right|^{2} \mathrm{~d} t\right)^{\frac{p}{4}}\right] \\
& \leq d_{p} E\left[\sup _{t \geq 0}\left|Y_{t}^{n}\right|^{\frac{p}{2}}\left(\int_{0}^{\infty}\left|Z_{t}^{n}\right|^{2} \mathrm{~d} s\right)^{\frac{p}{4}}\right]
\end{aligned}
$$

and thus

$$
\begin{aligned}
c_{p} E\left[\left|\int_{0}^{\infty} Y_{t}^{n} Z_{t}^{n} \mathrm{~d} W_{t}\right|^{\frac{p}{2}}\right] \leq \frac{1}{2} E & {\left[\left(\int_{0}^{\infty}\left|Z_{t}^{n}\right|^{2} \mathrm{~d} t\right)^{\frac{p}{2}}\right] } \\
& +\frac{d_{p}^{2}}{2} E\left[\sup _{t \geq 0}\left|Y_{t}^{n}\right|^{p}\right],
\end{aligned}
$$

where $d_{p}$ is a positive constant depending only on $p$. From (31) and (33), we have

$$
\begin{aligned}
E[ & \left.\left(\int_{0}^{\infty}\left|Z_{t}^{n}\right|^{2} \mathrm{~d} t\right)^{\frac{p}{2}}\right] \\
\leq & C_{p}\left[E\left[|\xi|^{p}\right]+\left(\int_{0}^{\infty} \gamma(t) \mathrm{d} t\right)^{\frac{p}{2}}\right] \\
& +C_{p}\left[1+\left(\int_{0}^{\infty} \gamma(t) \mathrm{d} t+\int_{0}^{\infty} \gamma^{2}(t) \mathrm{d} t\right)^{\frac{p}{2}}\right] E\left[\sup _{t \geq 0}\left|Y_{t}^{n}\right|^{p}\right],
\end{aligned}
$$

where $C_{p}$ is a positive constant depending only on $p$. Thus, there exists a constant $A>0$ independent of $n$, such that

$$
\begin{aligned}
& E\left[\sup _{t \geq 0}\left|U_{t}\right|^{p}\right] \leq A, \quad E\left[\left(\int_{0}^{\infty}\left|V_{t}\right|^{2} \mathrm{~d} t\right)^{\frac{p}{2}}\right] \leq A, \\
& E\left[\sup _{t \geq 0}\left|Y_{t}^{n}\right|^{p}\right] \leq A, \quad E\left[\left(\int_{0}^{\infty}\left|Z_{t}^{n}\right|^{2} \mathrm{~d} t\right)^{\frac{p}{2}}\right] \leq A, \quad \forall n \in N .
\end{aligned}
$$

The proof of Lemma 7 is complete.

Lemma $8 \quad\left\{\left(Y^{n}, Z^{n}\right)\right\}_{n=1}^{\infty}$ converges in $\mathcal{S}^{p}(R) \times \mathcal{L}^{p}\left(R^{d}\right)$.

Proof Since $\left\{Y^{n}\right\}_{n=1}^{\infty}$ is increasing and bounded in $\mathcal{S}^{p}(R)$, we deduce from the dominated convergence theorem that $Y^{n}$ converges in $\mathcal{S}^{p}(R)$. We shall denote by $Y$ the limit of $Y^{n}$. Applying Itô's formula to $\left|Y_{t}^{n}-Y_{t}^{m}\right|^{2}$, we get for any $n, m \in N$,

$$
\begin{aligned}
\mid Y_{0}^{n}- & \left.Y_{0}^{m}\right|^{2}+\int_{0}^{\infty}\left|Z_{t}^{n}-Z_{t}^{m}\right|^{2} \mathrm{~d} t \\
= & 2 \int_{0}^{\infty}\left(Y_{t}^{n}-Y_{t}^{m}\right)\left(g_{n}\left(t, Y_{t}^{n}, Z_{t}^{n}\right)-g_{m}\left(t, Y_{t}^{m}, Z_{t}^{m}\right)\right) \mathrm{d} t \\
& -2 \int_{0}^{\infty}\left(Y_{t}^{n}-Y_{t}^{m}\right)\left(Z_{t}^{n}-Z_{t}^{m}\right) \mathrm{d} W_{t} .
\end{aligned}
$$


Thus, we have

$$
\begin{aligned}
& \int_{0}^{\infty}\left|Z_{t}^{n}-Z_{t}^{m}\right|^{2} \mathrm{~d} t \\
& \quad \leq 2 \sup _{t \geq 0}\left|Y_{t}^{n}-Y_{t}^{m}\right| \int_{0}^{\infty}\left|g_{n}\left(t, Y_{t}^{n}, Z_{t}^{n}\right)\right| \mathrm{d} t+2 \sup _{t \geq 0}\left|Y_{t}^{n}-Y_{t}^{m}\right| \int_{0}^{\infty}\left|g_{m}\left(t, Y_{t}^{m}, Z_{t}^{m}\right)\right| \mathrm{d} t \\
& \quad+2\left|\int_{0}^{\infty}\left(Y_{t}^{n}-Y_{t}^{m}\right)\left(Z_{t}^{n}-Z_{t}^{m}\right) \mathrm{d} W_{t}\right| .
\end{aligned}
$$

Using the following fact: if $b, a_{i} \geq 0$ and $b \leq \sum_{i=1}^{n} a_{i}$, then $b^{p} \leq \sum_{i=1}^{n} a_{i}^{p}$ for any $p \in(0,1)$, it follows that

$$
\begin{aligned}
E\left[\left(\int_{0}^{\infty}\left|Z_{t}^{n}-Z_{t}^{m}\right|^{2} \mathrm{~d} t\right)^{\frac{p}{2}}\right] \\
\leq c_{p} E\left[\sup _{t \geq 0}\left|Y_{t}^{n}-Y_{t}^{m}\right|^{\frac{p}{2}}\left(\int_{0}^{\infty}\left|g_{n}\left(t, Y_{t}^{n}, Z_{t}^{n}\right)\right| \mathrm{d} t\right)^{\frac{p}{2}}\right] \\
\quad+c_{p} E\left[\sup _{t \geq 0}\left|Y_{t}^{n}-Y_{t}^{m}\right|^{\frac{p}{2}}\left(\int_{0}^{\infty}\left|g_{m}\left(t, Y_{t}^{m}, Z_{t}^{m}\right)\right| \mathrm{d} t\right)^{\frac{p}{2}}\right] \\
+c_{p} E\left[\left|\int_{0}^{\infty}\left(Y_{t}^{n}-Y_{t}^{m}\right)\left(Z_{t}^{n}-Z_{t}^{m}\right) \mathrm{d} W_{t}\right|^{\frac{p}{2}}\right],
\end{aligned}
$$

where $c_{p}$ is a positive constant depending only on $p$. From Schwarz's inequality, we have

$$
\begin{aligned}
& E\left[\sup _{t \geq 0}\left|Y_{t}^{n}-Y_{t}^{m}\right|^{\frac{p}{2}}\left(\int_{0}^{\infty}\left|g_{k}\left(t, Y_{t}^{k}, Z_{t}^{k}\right)\right| \mathrm{d} t\right)^{\frac{p}{2}}\right] \\
\leq & \left(E\left[\sup _{t \geq 0}\left|Y_{t}^{n}-Y_{t}^{m}\right|^{p}\right]\right)^{\frac{1}{2}}\left(E\left[\left(\int_{0}^{\infty}\left|g_{k}\left(t, Y_{t}^{k}, Z_{t}^{k}\right)\right| \mathrm{d} t\right)^{p}\right]\right)^{\frac{1}{2}}, k=n, m .
\end{aligned}
$$

By Lemma 6 (i), we can obtain

$$
\begin{aligned}
E[ & \left.\left(\int_{0}^{\infty}\left|g_{k}\left(t, Y_{t}^{k}, Z_{t}^{k}\right)\right| \mathrm{d} t\right)^{p}\right] \\
\leq & E\left[\left(\int_{0}^{\infty} \gamma(t)\left(1+\left|Y_{t}^{k}\right|+\left|Z_{t}^{k}\right|\right) \mathrm{d} t\right)^{p}\right] \\
\leq & d_{p}\left(\int_{0}^{\infty} \gamma(t) \mathrm{d} t\right)^{p}+d_{p}\left(\int_{0}^{\infty} \gamma(t) \mathrm{d} t\right)^{p} E\left[\sup _{t \geq 0}\left|Y_{t}^{k}\right|^{p}\right] \\
& +d_{p}\left(\int_{0}^{\infty} \gamma^{2}(t) \mathrm{d} t\right)^{\frac{p}{2}} E\left[\left(\int_{0}^{\infty}\left|Z_{t}^{k}\right|^{2} \mathrm{~d} t\right)^{\frac{p}{2}}\right], k=n, m
\end{aligned}
$$

where $d_{p}$ is a positive constant depending only on $p$. Thus, by Lemma 7 , there exists a constant $D>0$ independent of $n, m$ such that

$$
E\left[\sup _{t \geq 0}\left|Y_{t}^{n}-Y_{t}^{m}\right|^{\frac{p}{2}}\left(\int_{0}^{\infty}\left|g_{n}\left(t, Y_{t}^{n}, Z_{t}^{n}\right)\right| \mathrm{d} t\right)^{\frac{p}{2}}\right] \leq D\left(E\left[\sup _{t \geq 0}\left|Y_{t}^{n}-Y_{t}^{m}\right|^{p}\right]\right)^{\frac{1}{2}},
$$




$$
E\left[\sup _{t \geq 0}\left|Y_{t}^{n}-Y_{t}^{m}\right|^{\frac{p}{2}}\left(\int_{0}^{\infty}\left|g_{m}\left(t, Y_{t}^{m}, Z_{t}^{m}\right)\right| \mathrm{d} t\right)^{\frac{p}{2}}\right] \leq D\left(E\left[\sup _{t \geq 0}\left|Y_{t}^{n}-Y_{t}^{m}\right|^{p}\right]\right)^{\frac{1}{2}}
$$

By the Burkholder-Davis-Gundy inequality, we get

$$
\begin{aligned}
& c_{p} E {\left[\left|\int_{0}^{\infty}\left(Y_{t}^{n}-Y_{t}^{m}\right)\left(Z_{t}^{n}-Z_{t}^{m}\right) \mathrm{d} W_{t}\right|^{\frac{p}{2}}\right] } \\
& \leq e_{p} E\left[\left(\int_{0}^{\infty}\left|Y_{t}^{n}-Y_{t}^{m}\right|^{2}\left|Z_{t}^{n}-Z_{t}^{m}\right|^{2} \mathrm{~d} t\right)^{\frac{p}{4}}\right] \\
& \leq e_{p} E\left[\sup _{t \geq 0}\left|Y_{t}^{n}-Y_{t}^{m}\right|^{\frac{p}{2}}\left(\int_{0}^{\infty}\left|Z_{t}^{n}-Z_{t}^{m}\right|^{2} \mathrm{~d} t\right)^{\frac{p}{4}}\right]
\end{aligned}
$$

and thus

$$
\begin{aligned}
c_{p} E\left[\left|\int_{0}^{\infty}\left(Y_{t}^{n}-Y_{t}^{m}\right)\left(Z_{t}^{n}-Z_{t}^{m}\right) \mathrm{d} W_{t}\right|^{\frac{p}{2}}\right] \leq & \frac{1}{2} E\left[\left(\int_{0}^{\infty}\left|Z_{t}^{n}-Z_{t}^{m}\right|^{2} \mathrm{~d} t\right)^{\frac{p}{2}}\right] \\
& +\frac{e_{p}^{2}}{2} E\left[\sup _{t \geq 0}\left|Y_{t}^{n}-Y_{t}^{m}\right|^{p}\right],
\end{aligned}
$$

where $e_{p}$ is a positive constant depending only on $p$. From (37), (40), (41) and (43), we have

$$
\begin{aligned}
& E\left[\left(\int_{0}^{\infty}\left|Z_{t}^{n}-Z_{t}^{m}\right|^{2} \mathrm{~d} t\right)^{\frac{p}{2}}\right] \\
& \quad \leq C_{p}\left(\left(E\left[\sup _{t \geq 0}\left|Y_{t}^{n}-Y_{t}^{m}\right|^{p}\right]\right)^{\frac{1}{2}}+E\left[\sup _{t \geq 0}\left|Y_{t}^{n}-Y_{t}^{m}\right|^{p}\right]\right),
\end{aligned}
$$

where $C_{p}$ is a positive constant depending only on $p$. Thus, $\left\{Z^{n}\right\}_{n=1}^{\infty}$ is a Cauchy sequence in $\mathcal{L}^{p}\left(R^{d}\right)$, from which the result follows. The proof of Lemma 8 is complete.

Proof of Theorem 5 For all $n \in N$, we have $Y^{n} \leq U$, and $\left\{Y^{n}\right\}_{n=1}^{\infty}$ converges in $\mathcal{S}^{p}(R)$, $\mathrm{d} t \times \mathrm{d} P$-a.s. to $Y \in \mathcal{S}^{p}(R)$.

On the other hand, since $Z^{n}$ converges in $\mathcal{L}^{p}\left(R^{d}\right)$ to $Z$, we can assume, choosing a subsequence if needed, that $Z^{n} \rightarrow Z, \mathrm{~d} t \times \mathrm{d} P$-a.s., as $n \rightarrow \infty$ and $\bar{G}:=\sup _{n}\left|Z^{n}\right|$ is $\mathrm{d} t \times \mathrm{d} P$ integrable. Therefore, by Lemma 6 (i) and (iv), we get for almost all $\omega$,

$$
\begin{aligned}
g_{n}\left(t, Y_{t}^{n}, Z_{t}^{n}\right) \rightarrow g\left(t, Y_{t}, Z_{t}\right), \mathrm{d} t-\text { a.e., } \text { as } n \rightarrow \infty & \\
\left|g_{n}\left(t, Y_{t}^{n}, Z_{t}^{n}\right)\right| & \leq \gamma(t)\left(1+\left|Y_{t}^{n}\right|+\left|Z_{t}^{n}\right|\right) \\
& \leq \gamma(t)\left(1+\sup _{n}\left|Y_{t}^{n}\right|+\bar{G}_{t}\right) \in L^{1}([0, \infty) ; \mathrm{d} t) .
\end{aligned}
$$

Thus, for almost all $\omega$ and uniformly in $t$, it holds that 


$$
\int_{t}^{\infty} g_{n}\left(s, Y_{s}^{n}, Z_{s}^{n}\right) \mathrm{d} s \rightarrow \int_{t}^{\infty} g\left(s, Y_{s}, Z_{s}\right) \mathrm{d} s, \quad \text { as } n \rightarrow \infty
$$

From the continuity properties of the stochastic integral, it follows that

$$
\sup _{t \geq 0}\left|\int_{t}^{\infty} Z_{s}^{n} \mathrm{~d} W_{s}-\int_{t}^{\infty} Z_{s} \mathrm{~d} W_{s}\right| \rightarrow 0 \text { in probability, as } n \rightarrow \infty
$$

Choosing again, a subsequence, we can assume that the above convergence is $P$-a.s. Finally,

$$
\begin{aligned}
&\left|Y_{t}^{n}-Y_{t}^{m}\right| \leq \int_{t}^{\infty}\left|g_{n}\left(s, Y_{s}^{n}, Z_{s}^{n}\right)-g_{m}\left(s, Y_{s}^{m}, Z_{s}^{m}\right)\right| \mathrm{d} s \\
&+\left|\int_{t}^{\infty}\left(Z_{s}^{n}-Z_{s}^{m}\right) \mathrm{d} W_{s}\right|,
\end{aligned}
$$

and taking limits on $m$ and supremum over $t$, we get

$$
\begin{aligned}
\sup _{t \geq 0}\left|Y_{t}^{n}-Y_{t}\right| \leq \int_{0}^{\infty} & \left|g_{n}\left(s, Y_{s}^{n}, Z_{s}^{n}\right)-g\left(s, Y_{s}, Z_{s}\right)\right| \mathrm{d} s \\
& +\sup _{t \geq 0}\left|\int_{t}^{\infty}\left(Z_{s}^{n}-Z_{s}\right) \mathrm{d} W_{s}\right|, P-\text { a.s. }
\end{aligned}
$$

from which it follows that $Y^{n}$ converges uniformly in $t$ to $Y$ (in particular, $Y$ is a continuous process). Note that $\left\{Y^{n}\right\}_{n=1}^{\infty}$ is monotone; therefore, we actually have the uniform convergence for the entire sequence and not just for a subsequence. Taking limits in Equation (25), we deduce that $(Y, Z)$ is a solution of BSDE (2).

Let $(\tilde{Y}, \tilde{Z}) \in \mathcal{S}^{p}(R) \times \mathcal{L}^{p}\left(R^{d}\right)$ be any solution of BSDE (2). From Theorem 4, we get that $Y^{n} \leq \tilde{Y}, \forall n \in N$ and therefore $Y \leq \tilde{Y}$ proving that $Y$ is the minimal solution. The proof of Theorem 5 is complete.

Remark 9 By Theorem 5, we have: Under the assumption (A.3) and (A.4), for each given $\xi \in \mathcal{L}(\Omega, \mathcal{F}, P, R)$, BSDE (2) has a solution $(Y, Z) \in \mathcal{S}(R) \times \mathcal{L}\left(R^{d}\right)$. Also, in $\mathcal{S}(R) \times \mathcal{L}\left(R^{d}\right)$, there is a minimal solution $(\underline{Y}, \underline{Z})$ of BSDE (2), in the sense that for any other solution $(Y, Z)$ of (2), we have $\underline{Y} \leq Y$.

\section{Conclusion}

In this paper, we have solved two problems on infinite time interval BSDEs. Firstly, by using an a priori estimate (Lemma 3), we studied the existence and uniqueness theorem for $L^{p}(1<p<2)$ solutions of infinite time interval BSDEs with non-uniformly Lipschitz coefficients (Theorem 2). It should be pointed out that the assumptions of Theorem 2 is weaker than that of Theorem 3.1 in Zong (2013). Secondly, applying comparison theorem for 1-dimensional infinite time interval BSDEs (Theorem 4), we studied the existence theorem for $L^{p}(1<p<2)$ solutions of 1 -dimensional infinite time interval BSDEs under the conditions that the coefficients are continuous and have linear growths (Theorem 5). In Theorem 5, the existence of a minimal solution was also obtained. 


\section{Authors' contributions}

The authors contributed equally and significantly in writing this article. Both authors read and approved the final manuscript.

\section{Acknowledgements}

The authors would like to thank the anonymous referees for their constructive suggestions and valuable comments. This work was supported partly by the National Natural Science Foundation of China (Nos. 11301295, 11571198 and 11501319), the Education Department of Shandong Province Science and Technology Plan Project (Nos. J16LI07 and J15LI05), the Science and Technology Plan Project of Qufu Normal University (No. xkj201517), the Doctoral Foundation of Qufu Normal University, the Program for Scientific Research Innovation Team in Colleges and Universities of Shandong Province of China and the Program for Scientific Research Innovation Team in Applied Probability and Statistics of Qufu Normal University (No. 0230518).

\section{Competing interests}

The authors declare that they have no competing interests.

Received: 28 October 2015 Accepted: 28 September 2016

Published online: 06 October 2016

\section{References}

Briand P, Delyon B, Hu Y, Pardoux E, Stoica L (2003) $L^{p}$ solutions of backward stochastic differential equations. Stoch Process Appl. 108:109-129

Chen Z, Wang B (2000) Infinite time interval BSDEs and the convergence of $g$-martingales. J Aust Math Soc (Series A) 69:187-211

Darling RWR, Pardoux E (1997) BSDE with random terminal time and applications to semilinear elliptic PDE. Ann Probab 25:1135-1159

El Karoui N, Peng S, Quenez MC (1997) Backward stochastic differential equations in finance. Math Finance 7:1-7

Lepeltier JP, San Martin J (1997) BSDEs with continuous coefficients. Stat Probab Lett 32:425-430

Pardoux E (1997) Generalized discontinuous BSDEs. In: El Karoui N, Mazliak L (eds) Backward stochastic differential equations. Pitman Research Notes in Mathematics Series, vol 364. Harlow, Longman, pp 207-219

Pardoux E (1998) BSDEs, weak convergence and homogenization of semilinear PDEs. Nonlinear analysis, differential equations and control (Montreal, QC, 1998). Kluwer Academic Publishers, Dordrecht, pp 503-549

Pardoux E, Peng S (1990) Adapted solution of a backward stochastic differential equation. Syst Control Lett 14:55-61

Peng S (1990) A general stochastic maximum principle for optimal problems. SIAM J. Control Optim. 28:966-979

Zong Z (2013) $L^{p}$ solutions of infinite time interval BSDEs and the corresponding $g$-expectations and $g$-martingales. Turk J Math 37:704-718

\section{Submit your manuscript to a SpringerOpen ${ }^{\circ}$ journal and benefit from:}

- Convenient online submission

Rigorous peer review

- Immediate publication on acceptance

- Open access: articles freely available online

- High visibility within the field

- Retaining the copyright to your article

Submit your next manuscript at $>$ springeropen.com 